\title{
Admission Profile and Outcome of Patients in a Level III Intensive Care Unit: A Two-Year Comparative Study
}

\author{
Sunil Pathak, Subhash P Acharya, Pragya Acharya, Binita Bhattarai \\ Department of Anesthesiology, Maharajgunj Medical Campus, Tribhuvan University Teaching Hospital, Institute \\ of Medicine, Maharajgunj, Kathmandu, Nepal
}

\section{Corresponding author:}

Sunil Pathak, MBBS, MD

Department of Anesthesiology, Maharajgunj Medical Campus, Tribhuvan University Teaching Hospital, Institute of Medicine, Maharajgunj, Kathmandu, Nepal

Email: drpathak.sp@gmail.com

Submitted : Oct 4, 2020

Accepted : Dec 5, 2020

\begin{abstract}
\section{Introduction}

An Intensive Care Unit (ICU) is an area where critically ill patients are admitted and the highest level of care is provided with close intensive monitoring and management. Clinical audit is a must to measure indicators of the quality of care in ICU and benchmarking outcome. The main aim of the study was to evaluate the profile of patients admitted to ICU and assess their outcome of two consecutive years.
\end{abstract}

\section{Methods}

A single-institutional, descriptive cross-sectional study was conducted on all adult patients admitted to the ICU of Tribhuvan University Teaching Hospital, Kathmandu, between April 2018 and April 2020 (Baisakh 2075 to Chaitra 2076).

\section{Results}

With an average of 1.98 admissions per day, a total of 1447 patients were admitted to TUTH ICU over the period of two years. A male preponderance was noted with a male to female ratio of 1.19:1. Most of the patients admitted were neurosurgical cases 429 (29.6\%). A total of 884 (61\%) were shifted out of ICU in stable condition while the overall mortality was 458 (31.6\%).

\section{Conclusion}

This study shows that there is a higher severity of illness at admission as well as a comparatively high mortality rate. More number of patients were male, of age group of 15-65 years, with medical and neurosurgical conditions. This study of profile and outcome of patients admitted in ICU can serve as a quality indicator as well as evidence on which planning and policymaking can be based upon in the future.

\section{Keywords}

Intensive care unit, medical audit, outcome 


\section{INTRODUCTION}

ntensive care unit (ICU) is a multidisciplinary and inter-professional specialty where patients having, or at risk of developing acute, life-threatening organ dysfunction are managed. Intensive management is done by a group of skilled doctors and nurses with close and constant monitoring with highly sophisticated equipment. The patients are managed with various life supports like mechanical ventilation, hemodialysis etc. The ICU at Tribhuvan University Teaching Hospital (TUTH) is a semiclosed, eleven bedded mixed medical-surgical units.

An audit is a process of analyzing the data of the patient admitted during a certain time period in terms of profile and outcome to assess the quality of care by systematic review. ${ }^{1}$ It is used to monitor the progress in the healthcare delivery system. ${ }^{2}$ The present study was done to retrospectively compare the patient profile and outcome of two consecutive years which would provide the evidence upon which the planning and policy-making can be based in the future.

\section{METHODS}

This retrospective cross-sectional study reviewed all the patients, admitted to the intensive care unit of Tribhuvan University Teaching Hospital, Kathmandu, Nepal over a period of two years, from April 2018 to April 2020 (Baisakh 2075 to Chaitra 2076). Ethical approval was obtained from the Institutional Review Committee of the Institute of Medicine, Tribhuvan University. The ICU Admission Discharge Book, electronic medical record, and patient charts were reviewed and required data was extracted from it.

Data extracted from the records included age, sex, diagnosis, preoperative or postoperative, length of ICU stay, length of mechanical ventilation, and outcome. The outcome was classified as discharge, left against medical advice (LAMA), withdrawal from life support, and death. Data was analyzed using Microsoft Excel and the Statistical Package for Scientific Solutions (SPSS). Comparative data of two years were summarized using graphical methods.

\section{RESULTS}

A total of 1447 patients, admitted in the ICU over a period of two years, an average of 1.98 admissions per day; 767 and 680 in 2075 B.S and 2076 B.S respectively, were evaluated. There were total 789 (54.5\%) males and 658 (45.5\%) females. The majority of the patients belonged to the 15-65 years' age group with total number of 998 (68.9\%).

Out of 1447 patients, sequential organ failure assessment (SOFA) score at admission was <9 in $1077(74.5 \%)$ patients, 9 to 11 in 194 (13.4\%) and $>11$ in $176(12.1 \%)$ as shown in Figure 2.

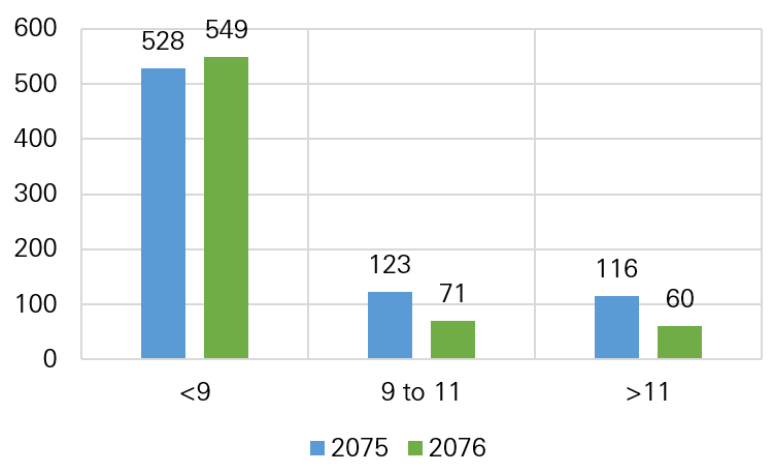

Fig 2. Patients distribution based on SOFA score on admission $(n=144)$

There were a total of $654(45.2 \%)$ medical patients and $793(54.8 \%)$ surgical patients. Amongst the department, maximum number of patients 429 (29.6\%) were admitted under neurosurgical department. The distribution based on primary specialty of admission is shown in Figure 1.

A total 968 patients (501 in 2075 and 467 in 2076 BS.) required mechanical ventilation (66.9\%). The

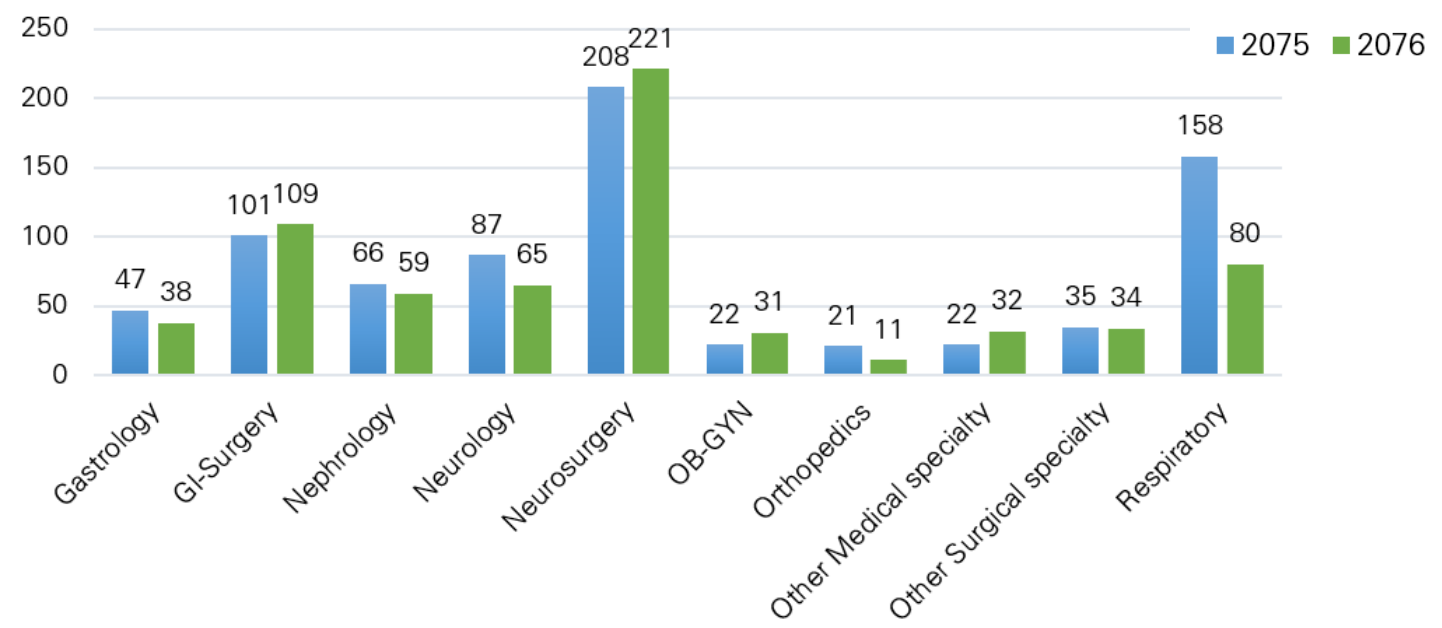

Fig 1. Distribution of patients admitted to ICU based on primary specialty $(n=144)$ 
Table 1. Outcome of patients in TUTH ICU

\begin{tabular}{lccc}
\hline \multicolumn{1}{c}{ Characteristics } & $\mathbf{2 0 7 4}$ & $\mathbf{2 0 7 5}$ & $\mathbf{2 0 7 6}$ \\
\hline Improved (\%) & 56.6 & 59.8 & 62.5 \\
Mortality (\%) & 33 & 33.1 & 30 \\
LAMA (\%) & 7.7 & 4.7 & 5.6 \\
Withdrawal (\%) & 2.7 & 2.4 & 1.9 \\
$\begin{array}{l}\text { Average length of } \\
\text { stay in MV (days) }\end{array}$ & 5.4 & 4.1 & 3.5 \\
$\begin{array}{l}\text { Average length of } \\
\text { stay in ICU (days) }\end{array}$ & 4.5 & 4.4 & 4.5 \\
\hline
\end{tabular}

average length of stay (days) in ICU was 4.4 and 4.5 respectively in each year of study duration. The average length of stay has remained fairly constant in the study period of two years.

The average length of stay (days) in mechanical ventilation (MV) was 4.1 and 3.5 respectively in the year 2075 and 2076 B.S. The average length of mechanical ventilation has improved owing to the improved practice of sedation vacation and weaning trials (Table 1).

The outcome of 1447 patients admitted in TUTH, ICU were analyzed which showed $884(61.1 \%)$ were improved and transferred out of ICU; 459 (59.8\%) in 2075 and $425(62.5 \%)$ indicating the increase in the rate of patients that improved and were transferred out from ICU. Out of 1447 admitted patients, 458 (31.7\%) patients died, 31 (2.1\%) patients underwent withdrawal of life support while $74(5.1 \%)$ patients were in the LAMA group due to various socio-economic reasons as shown in Figure 3. The rate of LAMA was $4.6 \%$ and $5.5 \%$ in 2075 and 2076 respectively, the rate is still high.

On analysis of age wise mortality, the results showed the number of mortality was 18 in those aged below 15 (26\% of total admission in the age group), 260 in those aged 15 to 65 years (26\% of total admission in the age group), and 180 in those aged more than 65 years $(47.2 \%$ of total admission in the age group.

Out of 884 patients shifted from ICU, most of them $576(65.1 \%)$ were transferred out to the Postoperative ward (POW) that is managed by Critical Care Outreach Team and is a level II step down unit. The remaining patients were transferred out of ICU to other step down units like Medical Intensive Care Unit (MICU), Surgical Intensive Care Unit (SICU) or to the floor under the care of primary admitting team.

\section{DISCUSSION}

The main objective of the audit is to see if the certain quality indicators are met so as to improve the outcome of the patients. ${ }^{1}$ The clinical audit seeks to improve patient care and outcomes

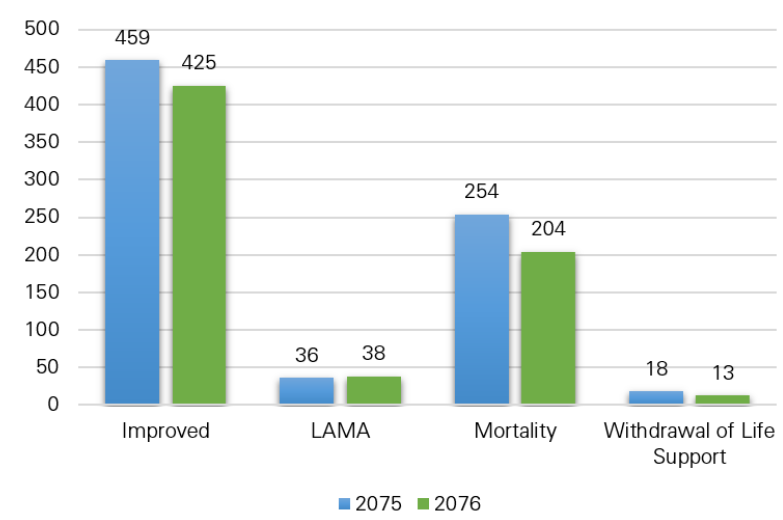

Fig 3. Distribution of patients admitted to ICU based on outcome $(n=144)$

through systematic review of care against explicit criteria and the implementation of change and further monitoring is used to confirm improvement in healthcare delivery." 2

Medical or health audit can be retrospective or operational. In retrospective audit, data of the patient admitted in certain ward is evaluated and analyzed after the patient is discharged from the unit. The operational audit is done by direct observation, interviews and document analysis during the period when the patient is admitted in the unit. ${ }^{3}$

The analysis of data revealed that $45.2 \%$ patients were admitted under medical specialty and $54.8 \%$ patients were admitted under surgical specialty with the ratio of 1:1.2. In the study conducted by Acharya et al. ${ }^{4}$ in the year 2074 in TUTH ICU admissions were predominantly medical (54.24\%) as shown in Table 2. This finding was different from the audit of other hospitals in Nepal; one study performed by Vaidhya et al. ${ }^{5}$ showed $72 \%$ were medical patients and $28 \%$ were surgical patients and in a study by Koirala et al. ${ }^{6}$ majorities (49\%) were surgical patients followed by medical (39\%). In another study by Cutherbertson et al. ${ }^{7}$ in an ICU, surgical patients made up $60-70 \%$ of the total admission. Amongst the surgical patients, neurosurgical patients were the most commonly admitted amounting to $54 \%$ of all surgical patients and $29.6 \%$ of all admission which had slightly increased from the year 2074 where neurosurgical patients accounted to $24.47 \%$ of all admission. ${ }^{4}$ As we have a comparatively high volume of neurosurgical patients, a separate

Table 2. Characteristics of the ICU admitted patients

\begin{tabular}{lccc}
\hline Characteristics & $\mathbf{2 0 7 4}$ & $\mathbf{2 0 7 5}$ & $\mathbf{2 0 7 6}$ \\
\hline Male (\%) & 51.8 & 55 & 54 \\
Female (\%) & 48.2 & 46 & 46 \\
Surgical (\%) & 45.8 & 50.5 & 59.7 \\
Medical (\%) & 54.2 & 49.5 & 40.3 \\
\hline
\end{tabular}


dedicated neuro-intensive care unit should be established in order to improve the quality of care of these patients.

In our study, out of 1447 patients, 789 (54.5\%) patients were male and 658 (45.5\%) patients were female with male to female ratio of 1.2:1 which was almost similar to the study conducted by Acharya et al. ${ }^{4}$ with the ratio of 1.07:1. The gender profile of the patients were also similar to the study by Vaidya et al. ${ }^{5}$ and Isamade et.al. ${ }^{8}$

A total of 884 (61\%) were improved and transferred out from ICU, which was higher than in the year 2074 where 460 (56.5\%) of total admitted patients were improved and transferred out from ICU. ${ }^{4}$ However, this data is slightly lower than other studies in the country where discharge rate was $63 \%$ in study by Vaidhya et al and $61 \%$ in study by Koirala et.al. 5,6

There were 458 deaths in the ICU during the study period of two years accounting to $31.7 \%$ of total admissions. This indicates a slight improvement in mortality rate from $33 \%$ in the year $2074 .{ }^{4}$ The mortality rate is lower compared to other studies in other developing countries where mortality ranged from 35.1 to $61.4 \% .^{8-10}$ However, mortality was higher compared to other studies conducted in the

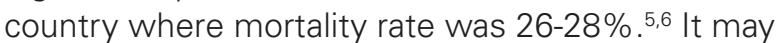
be because our hospital is the tertiary referral center of the country and very sick patients from all over the country are referred here. Out of 1447 patients, $370(25.6 \%)$ had SOFA score of $\geq 9$ at admission which could have explained the higher mortality in our ICU. As our center is a tertiary referral center, timely referral can help in improving the mortality rate. Also, improving the nurse patient ratio to $1: 1$, adequate training to the ICU nurses, strict adherence to the hand hygiene, helps in improving the outcome of patients admitted in ICU.

On evaluating the age-wise data, mortality was highest among aged more than 65 with 47.2\% of total admission which was higher than the year 2074 accounting for $41.37 \% .{ }^{4}$ The outcome of patients primarily depends on the severity of illness at admission which can be calculated using SOFA score. It also depends on the course of illness progression during the stay, hospital acquired infections and any critical incidents. The documentation of critical incidences, known as Critical Incident Reporting System is not very well done in our settings due to lack of proper electronic medical system and proper data recording facility. Sometimes patients belonging to lower socioeconomic status who could be salvaged if given critical services can't afford the treatment; thereby affecting the outcome.

During the two-year study period, 74 (5.1\%) patients were taken from the hospital against medical advice (LAMA), which is a significant number. Although the rate of patients leaving against medical advice has improved slightly when compared to the year 2074 $(7.7 \%)$, it continues to remain high. ${ }^{4}$ This data was also comparable to studies in the country by Vaidhya et.al and Koirala et al. ${ }^{5,6}$ The most common reason for LAMA is attributed to the financial burden to the family due to the lack of proper health insurance or security in the country as patients' family are supposed to bear all the expenses. The provision of health insurance or security from the government can help to improve this data. The rate of LAMA was only $0.2 \%$ of total admission in a study by Poluyi et.al conducted in Uganda. ${ }^{10}$

Out of 884 patients shifted from ICU, most of them $576(65.1 \%)$ were transferred out to the postoperative ward (POW). The remaining patients were discharged out of ICU to other step down units or to the floor under the care of primary admitting team.

The main limitation of this study was that the data were extracted from the ICU admission and discharge register, there might have been some errors if the data were not entered properly. Also long term follow-up of patients after getting shifted out of ICU was missed. The implementation of Electronic Medical Record system has been the need of today in order to have better audit in future.

\section{CONCLUSION}

The most number of patients admitted in ICU were of male gender, age group of 15-65 years with medical and neurosurgical conditions. The mortality remained constant over the years of study period but with comparatively high mortality rate.

\section{CONFLICT OF INTEREST}

None declared.

\section{ACKNOWLEDGEMENTS}

We would like to acknowledge all the medical officers and nursing staff in TUTH ICU, who during their regular clinical duties, contributed significantly in data recording process.

\section{REFERENCES}

1. Limb C, Fowler A, Gundogan B et al. How to conduct a clinical audit and quality improvement project? Int J Surg Oncol (N Y). 2017 Jul;2(6):e24

2. National Institute of Health and Care Excellence. Available at https://www.nice.org.uk/.

3. Bernardo L, Bernardo G, de Araujo R et al. The Auditing Process in Intensive Care Unit: A Systematic Review. ACRI. 2018;13(1):1-3.

4. Acharya SP, Bhattarai A, Bhattarai B et al. An Audit of An Intensive Care Unit of A Tertiary Care Hospital. J Nepal Med Assoc. 2018;56(212):759-762.

5. Vaidya PR, Shrestha RR. Outcome of the Patients Admitted in New Intensive Care Unit, Bir Hospital: Five years' review. PMJN. 2015;15(1):1-5.

6. S Koirala, A Ghimire, A Sharma et al. ICU admission and outcomes 
in a community-based tertiary care hospital: an audit of one year. Health Renaissance. 2011;9(2):83-87.

7. Cuthbertson BH, Webster NR. The role of the intensive care unit in the management of the critically ill surgical patient. J.R.Coll.Surg. Edinb. 1999;44:294-300.

8. Isamade ES, Yiltok SJ, Uba AF et al. Intensive care unit admissions in the Jos University Teaching Hospital. Nigerian J Clinical Practice. 2007;10:156-161.
9. Abubakar A, E Ojo, El-Nafaty A et al. An Audit of One-Year Intensive Care Practice In A Developing Country. The Internet Journal of Anesthesiology. 2007;18(2).

10. Poluyi EO, Fadiran 00, Poluyi CO et al. Profile of Intensive Care Unit Admissions and Outcomes in a Tertiary Care Center of a Developing Country in West Africa: A 5 Year Analysis. J Intensive Crit Care. 2016;2:1-7. 